\title{
Pelvic inflammatory disease, correlation between clinical and laparoscopic diagnosis - A review.
}

\author{
Nyengidiki K Tamunomie \\ Senior lecturer, University of Port Harcourt Teaching Hospital, Port Harcourt, Department of Obstetrics and \\ Gynaecology, Rivers State, Nigeria
}

\begin{abstract}
Background: Ascending infections of the upper genital tract are associated with morbidities such as infertility, chronic pelvic abscess, chronic pelvic pain, and ectopic pregnancy, if not treated properly. The treatment of pelvic inflammatory disease requires accurate diagnosis which largely is dependent on laid out clinical criteria that had been noted to lack specificity. Laparoscopy has been used as a gold standard over time but is not used routinely in the diagnosis of this disease entity.

Objective: The objective was to determine the correlation between use of clinical diagnostic criteria and laparoscopy in the diagnosis of pelvic inflammatory disease.

Design/Method: An electronic literature search of articles published on the subject from 1969 to 2012, using search engines: Highwire, Springerlink, Hinari, Pubmed, and Google were analyzed.

Outcome: There is poor correlation between clinically diagnosed pelvic inflammatory disease and laparoscopic confirmations resulting in over diagnosis of the disease. Conclusion: Clinical criteria for the diagnosis of pelvic inflammatory disease have a low predictive value. The routine use of laparoscopy appears to be a better option to reduce over diagnosis of this condition, missed diagnosis of other pelvic pathology, and ultimately to reduce expenditure incurred in overtreatment.
\end{abstract}

Key words: Clinical criteria, laparoscopy, pelvic inflammatory disease

\section{Introduction}

Pelvic inflammatory disease is an infection of the upper genital tract involving all or either of the following structures: cervix, uterus, fallopian tubes, ovaries, and its surrounding structures. ${ }^{1-3}$

There are several risks factors associated with this condition among which are multiple sexual partners, unsafe termination of pregnancies, uterine instrumentation, no previous pregnancy, non use of barrier contraceptive method, intrauterine contraceptive device, early coitache, past history of pelvic inflammatory disease and tobacco smoking, previous stillbirths, and multiple sexual partners. ${ }^{[4-7]}$

Its improper diagnosis and treatment is a cause of medical and socioeconomic sequelae: It constitutes about $2 \%$ of gynaecological consultations of young women to their primary care physician in England and Wales. ${ }^{[8]}$ Adolescents and young adults account for nearly half of the 780,000 cases of PID reported annually in the United States with 250,000 women hospitalized annually and 18/10,000 hospital discharges. Huge budgetary expenditure on its treatment is found in the USA where about 5.5 billion dollars are spent annually on its treatment and sequelae. ${ }^{[9,10]}$ In a resource-poor setting it accounts for $17-40 \%$ of gynecological admissions in Sub-Saharan Africa, 15-37\% in South East Asia, and 3\% in India. ${ }^{[11]}$

PID has been associated with chronic pelvic pain, tubovarian abscess and ectopic pregnancies, menstrual abnormalities, and sexual dysfunction. ${ }^{[12]}$ Tubal pathology resulting in infertility due to PID range from $35 \%$ in developed to $85 \%$ in developing countries with resultant increase in the rate of ectopic pregnancies - a sevenfold increase in rate as against those without ectopic pregnancy. ${ }^{[13,14]}$

\section{Etiology}

The main microbial organism implicated in the cause of PID is Chlamydia trachomatis, $\mathrm{N}$ gonorrhea and organisms associated with bacterial vaginosis. ${ }^{[15-17]}$ Following the primary infection a milieu is formed for other aerobic and anaerobic organisms to produce a secondary infection by staphylococcal spp., streptococcal spp., coliforms, and hemophilus influenza organisms. Anaerobes such as fusobacterium, peptococus, and variety of clostridium organisms have also been implicated. ${ }^{[1,18,19]}$ The increase in the prevalence of HIV has resulted in a higher incidence of mycobacterium tuberculin bacilli isolated in endometrial samples of patients with PID. ${ }^{[20]}$ Since the 1970s, the overall incidence of gonococcal infections had reduced with a corresponding increase in the incidence of Chlamydia infections in both developing and developed countries, this is as a result of increased awareness of the disease and the availability of more sensitive testing methods. ${ }^{[21-23]}$ 


\section{Route of infection/pathology}

Most cases of pelvic inflammatory disease are due to ascending infections of the genital tract transversing the endocervix into the uterus, fallopian tubes, and adjourning structures of the uterus. Only 10 $20 \%$ of lower reproductive tracts infections organisms ascend into the upper genital tract to cause disease and even smaller percentage of women with PID develop chronic sequelae. Microbes can be introduced during sexual intercourse or during cervical instrumentation procedures. The response of the host immune system and the type of organism determine the range of clinical manifestations of the disease condition resulting in either or all of the following: cervicitis, endometritis, salpingitis, salpingo-oophoritis, tubovarian abscess, pelvic peritonitis or abscess formation. ${ }^{[1,23]}$

\section{Diagnosis of pelvic inflammatory disease -- clinical features vs. laparoscopy}

Variations in the etiology and the immune responses of host to the causative organisms makes features of this disease non-pathognomonic with each clinician developing independent diagnostic criteria. This poses a clinical challenge to clinicians resulting in missed diagnosis of other clinical disease entities or an over diagnosis of PID. The commonly used diagnostic protocol was that developed by Jacobson and Westron in 1969 which tags a patient with PID, if acute lower abdominal pain/pelvic pain is accompanied by symptoms/signs of either two of the following: abnormal vaginal bleeding, menstrual anomalies, proctitis, fever, vomiting urinary symptoms, marked pelvic tenderness palpable abdominal mass or swelling with an erythrocytes sedimentation rate of $>15 \mathrm{~mm} / \mathrm{h} .{ }^{[24]}$ In order to further improve on the specificity of the clinical diagnosis criteria agencies such as the Centre for Disease and Control ${ }^{[25]}$ had modified the diagnostic criteria of Jacobson specifying three minimum diagnostic criteria identified on pelvic examination as

- lower abdominal pain

- cervical motion tenderness

- uterine tenderness/adnexae tenderness.

One or more of the following additional criteria can be used to enhance the specificity of the minimum criteria and support a diagnosis of PID:

- oral temperature $>101^{\circ} \mathrm{F}\left(>38.3^{\circ} \mathrm{C}\right)$;

- abnormal cervical or vaginal mucopurulent discharge;

- presence of abundant numbers of WBC on saline microscopy of vaginal fluid;

- elevated erythrocyte sedimentation rate

- elevated C-reactive protein

- laboratory documentation of cervical infection with $N$. gonorrhea or C. trachomatis.

A large multisite U.S. study found that using the adnexae tenderness as the minimal criteria raised the sensitivity of the Centre for Disease Control and prevention criteria from 83\% to 95\% and that even the modified 2002 CDC criteria failed to identify the women with subclinical PID who are at the same risk of having long-term sequelae if their disease is not treated. ${ }^{[2,26]}$

Thus despite the formulations of various guidelines, the diagnostic criterion of pelvic inflammatory diseases still remains an enigma. In order to overcome this diagnostic clinical dilemma, standard findings at laparoscopy were adopted as the "gold standard" for its diagnosis ${ }^{[24]}$ However its cost, requirement of general anesthesia, need for trained medical personnel, and nonvisualization of some areas of the uterus had impaired its utilization; especially in developing countries where diagnosis is still largely based on unspecific clinical features. ${ }^{[27,28]}$ Laparoscopic criteria for visual diagnosis of acute pelvic inflammatory disease include marked hyperemia of the tubal surface, edema of the tubal wall, and sticky exudate on the surface of the tube and fimbriae ends when open. ${ }^{[24]}$ These criteria are helpful in identifying sequelae of chronic pelvic inflammatory disease such as pyosalpinx which is diagnosed when tubes are enlarged, edematous with partial or total destruction of the fimbriated end.

\section{Materials And Methods}

An electronic search of literature concerned with diagnosis of pelvic inflammatory disease using clinical criteria and laparoscopy and other related information was conducted. The period of interest was from 1968 to 2012 using the search engines: Highwire, Pubmed, Springerlink, and Google. The results of the search were collated in a tabular form and the predictive values calculated in percentage.

\section{Results}

The results of the electronic search are as shown in Table 1:

Results were collated in terms of the authors, sample size, number of patients diagnosed clinically, and the corresponding number confirmed by laparoscopy and predictive value calculated in percentage. Results and recommendations of investigators were also examined. 
Table 1: Studies comparing percentage of accuracy of clinically diagnosed PID vs. laparoscopically confirmed disease

\section{Authors}

Jacobson et al., 1969 $9^{[24]}$

Chaparro et al., $1978^{[29]}$

Burchell et al., 1987 $7^{[30]}$

Hadgu et al., 1987 $7^{[31]}$

Westrom et al., 1992 ${ }^{[32]}$

Marcos et al., 1993 ${ }^{[33]}$

Eschenbach et al., 155 $1997^{[34]}$

Molander et al., 2000 33

Cibila et al., 2001 ${ }^{36]} \quad 141$

Laiama et al., $2009^{[37]} \quad 73$

Total no. of patients $\quad 4784$
Clinically diagnosed

814

223

40

628

2501

76

3

1

$\begin{aligned} & \text { Laparoscopically } \\ & \text { diagnosed }\end{aligned}$
532
103
22
414
1884
134
127
20
44
52
3332

Percentage of accuracy

(\%)

65.4

46.1

55

65.9

75

76.1

82

60.1

31.2

71.2

69.6

\section{Discussion}

The overall positive predictive value (PPV) of a clinical diagnosis of PID was 69.7\% when confirmed by laparoscopy which implied that $30.3 \%$ of patients were wrongly diagnosed for pelvic inflammatory disease because of the nonspecific nature of the clinical diagnostic criteria. In their series of 814 cases, Jacobson et al. had 532 cases of suspected PID confirmed using laparoscopy, 12\% had other pathological conditions, and 23\% had no obvious pathology. The essence of early diagnosis and treatment was noted in the finding of increased potential of tubal patency in patients identified and treated before adnexae mass or tenderness was diagnosed clinically. ${ }^{[24]}$

In support of the laparoscopic diagnosis, Simm et al. ${ }^{[38]}$ found a low specificity and sensitivity for commonly used clinical features such as abnormal vaginal discharge, irregular vaginal discharge, marked tenderness on bimanual examination, and ESR $>15 \mathrm{~mm} / \mathrm{h}$. There was little variation observed in the likelihood ratio and post-test probabilities among the variables studied. The discriminary analysis showed that three indexes largely influenced the prediction of PID, i.e., ESR $(P<0.001)$, fever $(P<0.0001)$, and adnexae tenderness $(P<0.0001)$.

Similar studies by Burchell and Schoon confirmed clinically diagnosed PID by laparoscopy in 55\% of the patients and used same method in differentiating other life-threatening conditions to the patient like ectopic pregnancy and acute appendicitis in $15 \%$ of patients who would have been missed by the use of clinical diagnostic criteria. In addition, they confirmed the superiority of laparoscopically assisted microbiological specimens against the vaginal specimen. ${ }^{[30]}$

Furthermore, Morcos et al. confirmed a predictive value of $76.1 \%$ for clinical diagnosis of PID. They identified that the optimal set of PID indicators consisting of adnexae tenderness, lower abdominal pain of less than 1week duration, and an elevated white cell count had sensitivity and specificity of $86.6 \%$ and $45.7 \%$ respectively with estimated predictive values for positive and negative tests being 0.84 and 0.52 respectively. They advised a must use of laparoscopy in the diagnosis of PID. ${ }^{[33]}$

Cibula et al. in their prospective analysis of patients with acute recurrent PID clinically diagnosed PID confirmed diagnosis in 30\% of patients. Adhesions without features of PID were found in $16 \%$ of patients and they suggested that cases of PID recurrence and laboratory findings of such should be confirmed or excluded by laparoscopy. ${ }^{[36]}$

Using accepted clinical diagnostic criteria, Ieima et al. did a prospective study of 73 clinically diagnosed patients with PID and had a predictive value of $71.2 \%$. This finding suggested that laparoscopy improved the diagnosis of pelvic inflammatory disease and determined the severity of the clinical condition though sensitivity for milder cases may be lower. ${ }^{[37]}$

Some investigations have questioned the use of laparoscopy in making a diagnosis. Sellors et al. demonstrated $50 \%$ sensitivity and $85 \%$ specificity if laparoscopy is compared with histological analysis of fimbriael samples and Eckert et al. demonstrated that in 152 cases of clinically diagnosed PID, 26 had histological diagnosis of endometritis without laparoscopic evidence. ${ }^{[39,40]}$ Nevertheless the clinical application of these modes of diagnosis is largely impracticable and may worsen the patients' clinical state hence the need for supplementary much lesser hazardous intervention.

One of the reasons put forward by opponents of the routine use of laparoscopy is the financial implications of its use; however, Method et al., in a review of the costs of hospitalization, treatment and the inherent diagnostic error rate of clinically diagnosed patients, found no significant additional expense if all the women admitted with a clinical diagnosis of PID had had laparoscopic verification of their clinical diagnoses compared to the 
cost of treatment with intravenous antibiotics for 3-5 days and thus recommended the early use of laparoscopy in establishing the diagnosis of PID because it ensures a more accurate and definitive diagnosis and does not add significantly to costs. ${ }^{[41]}$

\section{Conclusion/Recommendations}

Based on the nonspecific nature of several clinical diagnostic criteria and low predictive value for pelvic inflammatory, the use of laparoscopy in the diagnosis of this clinical condition appeared justified. Its use will facilitate the identification of life-threatening gynecological conditions that may be missed and also permit carrying interventional therapy for patients having tubovarian masses and abscess as part of the spectrum of disease entity.

\section{References}

[1]. Nazar NA, Anthony G. Pelvic inflammatory disease. In: Robert Davids, Ash L, edited. Gynaecology. $4^{\text {th }}$ ed. Church Livingstone, Elsevier Publishers; 943-954.

[2]. Wiesenfiled HS, Sweet RL, Ness RB, Krohn MA, Amotegni AJ, Hiller SL. Comparison of acute and subclinical Pelvic inflammatory disease. Sex Transm Dis 2005;32:400-5.

[3]. Alison SB. Pelvic Inflammatory disease. ObstetGynaecol 2004; 6:138-44.

[4]. Frei BJ. Acute Pelvic inflammatory disease. Semin Adolesc Med 1986;2:143-53.

[5]. Gogate A, Rabin S, Nicholas S, Gogate S, Gaonkar T, Naidu A, et al. Risk factors for laparoscopically confirmed pelvic inflammatory disease: Findings from Mumbai(Bombay ) India. Sex Transm Inf 1998;74:426-32.

[6]. Grosestein F, Rothman KJ. Epidemiology of Pelvic inflammatory disease. Epidemiology 1994;5:234-42.

[7]. Simms I, Rogers P, Charlett A. The rate of diagnosis and demographics of Pelvic inflammatory disease in general practice England and Wales. Int J STD AIDS 1999;10:448-51.

[8]. Washington AE, Goves S, Schachter J, Sweet RL. Oral contraception, Chlamydia trachomatis infection and Pelvic inflammatory disease: A word of caution about protection. JAMA 1985;253:2246-50.

[9]. Rein DB, Kassler WJ, Irwin KL, Rabiee L. Direct medical cost of pelvic inflammatory disease and its sequelae: Decreasing but still substantial. Obstet Gynecol 2000;95:397-402.

[10]. Washington AE, Katz P. Cost of and payment source for Pelvic inflammatory disease, trends and projections 1993 through 200. JAMA 1991;266:2565-9.

[11]. Kani J, Adler MW. Epidemiology of Pelvic disease. In: Bergers GS, Westrom L, editors. Inflammatory diseases. New York: Raven press; 1992.

[12]. Jaiyeoba O, Soper DE. A practical approach to the diagnosis of Pelvic inflammatory disease. Infect Dis Obstet Gynecol 2011;2011:753037.

[13]. World Health Organization. Infections, pregnancy and infertility: Perspectives in prevention. Fertil Steril 1987;47:964-8.

[14]. Westrom L, Bengtoson LP, Mardh PA. Incidence, trends and risk of ectopic pregnancy in a population of women. Br Med J 1981;182:15-8.

[15]. Jorma P, Matti L. Chlamydia Pelvic inflammatory disease. Human Reproduction Update 1996 2:529-.

[16]. Soper DE, Brockwell NJ, Dalton HP, Johnson D. Observations concerning the microbial aetiology of acute salpingitis. Am J Obstet Gynecol 1994;170:1008-14.

[17]. Heinonen PK, Miettinen A. Laparoscopic study of the microbiology and severity of acute pelvic inflammatory disease. Eur J Obstet Gynaecol Reprod Biol 1994;57:85-9.

[18]. Chow AW, Malkascan KL, Marshall JR, Guze LB. The bacteriology of acute pelvic inflammatory disease. Am J Obstet Gynecol 1975;122:876-9.

[19]. Viberga I, Odline C, Lazdane G,Kroka J, Bergland L, Oloffsson S. Microbiology profile of women with Pelvic inflammatory disease in relation to IUD use. Infect Dis Obstet Gynecol 2005;13:183-90.

[20]. Avan BI, Fatmi Z, Rashid S. Comparison of clinical and laparoscopic features of infertile women suffering from genital tuberculosis or Pelvic inflammatory disease or endometriosis. J Pak Med Assoc 2001;51:393-9.

[21]. Poku BK, Sarkodie Y. Prevalence of genital Chlamydia and gonococcal infections in at risk women in the Kumasi metropolis, Ghana. Ghana Med J 2010 44:21-4.

[22]. Alison M, James SB. Pelvic Inflammatory. The Obstet Gynaecol 2004;6:138-44.

[23]. Ross J. Recent advances in the management of Pelvic inflammatory disease. European Genito-urinary disease 2007;2:33-5.

[24]. Jacobson L, Westrom L. Objective diagnosis of acute pelvic inflammatory disease. Diagnostic and prognostic value of routine laparoscopy. Am J Obstet Gynecol 1969;105:1088-98.

[25]. Centre for the disease control and prevention. Sexually transmitted disease treatment guidelines 2002. MMWR 2002; 51:48-52.

[26]. Piepert JF, Ness RB, Blume J, Soper DE, Holley R, Randall H, et al. Clinical prediction of endometritis in women with symptoms and signs of pelvic inflammatory disease. Am J Obstet Gynecol 2001;184:856-63.

[27]. Paavonen J. Pelvic inflammatory disease from diagnosis to prevention. Dermatol Clin 1998 16:747-56.

[28]. Fawole AO, Awonuga DO. Gynaecological emergencies in the Tropics. Recent advances in the management. Annuals of Ibadan Post Graduate Medecine 2007 Vol 5:12-20.

[29]. Chaparro MV, Gosh S, Nashed A, Poliak A. Laparoscopy for the confirmation of and prognostic evaluation of pelvic inflammatory disease. Int J Gynaecol Obstet 1978:15:307-91.

[30]. Burchell HJ, Schoon MG. The value of laparoscopy in the diagnosis of acute pelvic inflammatory disease. S Afr Med J 19871;72:197-8.

[31]. Hague A, Westron, L brookes CA, Reynold GH, Thompson SE. Predicting acute pelvic inflammatory disease a multivariate analysis. Am J Obstet Gynecol 1986;155:954-50.

[32]. Westrom L, Joestoet R, Reynolds G, Hagdu A, Thompson SE. Pelvic inflammatory disease and fertility. A cohort study of 1,844 women with laparoscopically verified disease and 657 control women with normal laparoscopic results. Sex Transm Dis 1992;19:185-92.

[33]. Morcos R, Frost N, Hnat M, Petrunak A, Caldito G. Laparoscopic vs clinical diagnosis of acute pelvic inflammatory disease. J Reprod Med 1993;38:53-6. 
[34]. Eschenbach DA, Wolner-Hanssen P, Hawes SE, Palvetic A, Paavonen J, Holmes KK. Acute inflammatory disease association of clinical and laboratory findings with laparoscopic findings. Obstet Gynecol 1997;89:184-92.

[35]. Molander P, Cacciatore B, Sjoberg J, Paavonen P. laparoscopic management of suspected acute pelvic inflammatory disease. J Am Assoc Gynecol laparosc2000;7:107-10.

[36]. Cibila D, Kuzel D, Fuakora Z, Svabik K, Zivny J. Acute exacerbation of recurrent pelvic inflammatory disease laparoscopic findings in 141 women with clinical diagnosis. J Reprod Med2001;46:49-53.

[37]. Maleckiene L, Kajenas S, Nadisauskiene RJ, Railaite DR. Comparison of clinical and laparoscopic diagnosis of pelvic inflammatory disease. Int J Gynaecol Obset 2009 10:74-5.

[38]. Simms I, Warburton F, Westrom L. Diagnosis of Pelvic inflammatory disease: Time for a rethink. Sex Transm Infect 2003;79:491 4.

[39]. Sellors JW,Mohoney JB, Goldsmith C, Rath D, Mander R, Hunter B, et al. The diagnosis of Pelvic inflammatory disease: The accuracy of clinical and laparoscopic findings. Am J Obset Gynaecol1991;164:113-20.

[40]. Eckert LO, Hawes ES, Woher-Hanseen PK, Kiviat NB, Wasserheit JN, Paavonen JA, et al. Endometritis, the clinical pathologic syndrome. Am J Obstet Gynecol2002;186:690-5.

[41]. Methods MW, Urnes PD, Neahring R, Sciarra JJ, Keith LG. Economic considerations in the use of laparoscopy for the diagnosis of Pelvic inflammatory disease. J Reprod Med 1987;32:759-64. 